\title{
VISUAL TARGET PROCESSING IN HIGH- AND LOW PERFORMING OLDER SUBJECTS INDEXED BY P3 COMPONENT
}

\section{LE P3 EN TANT QU'INDEX DU NIVEAU DE PERFORMANCE DE SUJETS ÂGÉS IMPLIQUÉS DANS UNE TÂCHE VISUELLE}

\author{
Authors: L. Lorenzo-López*, E. Amenedo, P. Pazo-Álvarez, F. Cadaveira
}

This is the peer reviewed version of the following article: Lorenzo-López, L., Amenedo, E., PazoÁlvarez, P., Cadaveira, F. (2007). Visual target processing in high- and low-performing older subjects indexed by P3 component. Neurophysiologie Clinique / Clinical Neurophysiology, 37, 53-61. doi: 10.1016/j.neucli.2007.01.008

This article may be used for non-commercial purposes in accordance with Elsevier, International Federation of Clinical Neurophysiology, Italian Clinical Neurophysiology Society, Japanese Society of Clinical Neurophysiology, Brazilian Society of Clinical Neurophysiology terms and conditions for use of self-archived versions. 


\title{
Visual target processing in high- and low-performing older subjects indexed by P3 component
}

\section{Le P3 en tant qu'index du niveau de performance de} sujets âgés impliqués dans une tâche visuelle

\author{
Authors:L. Lorenzo-López*, E. Amenedo, P. Pazo-Álvarez, F. Cadaveira
}

Department of Clinical Psychology and Psychobiology, Faculty of Psychology, University of Santiago de Compostela, Galicia, Spain

*Corresponding author: laurall@usc.es (L. Lorenzo-López). 


\begin{abstract}
Aim. - To explore the possible changes in the parameters of the P3 event-related potential (ERP) component among groups of young and older healthy subjects characterized as either high- or low-performers in a visual attention task.
\end{abstract}

Methods. - Both conventional and single-trial analyses of the visual P3 component were performed on each group of subjects.

Results. - P3 component significantly increased in latency as a function of age. The high performing older subjects showed the posterior predominance of P3, as in young subjects. However, the low-performing older subjects showed a significant P3 amplitude reduction at posterior locations and topographically more widespread activity. Furthermore, single-trial analysis showed that low-performing older subjects presented higher intertrial variability in P3 latency, few trials with P3 generation, and a reduced P3 amplitude in these trials in whom P3 was generated.

Conclusion. - These data suggest a specific decline in visual target processing in the low performing older subjects, which would imply a reduction in these attentional brain resources that are allocated to correctly select the relevant stimuli. The implications of this finding for the actual compensation versus dedifferentiation debate in normal aging are discussed.

Résumé

But. - Comparer les paramàtres du composant P3 des potentiels évoqués dans un groupe de sujets jeunes en bonne santé et dans un groupe de sujets âgés classifiés en fonction de leur niveau d'exécution d'une tâche d'attention visuelle (meilleurs et moins bons exécuteurs).

Méthodes. - Des analyses conventionnelles et des analyses « en sweep unique » du composant P3 ont été réalisées dans chaque groupe de sujets.

Résultats. - Le temps de latence du composant P3 augmente de fac son significative avec l'âge. Chez les sujets âgés meilleurs exécuteurs le P3 prédominait au niveau des régions postérieures, comme chez les sujets jeunes. Par contre, chez les sujets âgés moins bons exécuteurs, le P3 était significativement moins ample en postérieur et plus diffusément réparti sur le scalp. Les analyses «en sweep unique » ont montré que ces derniers présentaient une plus grande variabilité interessai en ce qui concerne le temps de latence de P3, moins de tests où le P3 était présent, ainsi qu'une réduction de l'amplitude de $\mathrm{P} 3$ dans les tests où il était présent.

Conclusion. - Ces données suggèrent l'existence d'un déficit du traitement visuel des stimuli chez les sujets âgés moins bons exécuteurs qui pourrait consister en une réduction des ressources 
cérébrales attentionnelles mobilisées pour sélectionner correctement le stimulus approprié. Nous discutons les implications de ces résultats dans le d'ebat concernant la redistribution des aires corticales actives chez les sujets âgés (hypothèses de compensation versus dédifférentiation). 


\section{Introduction}

The P3 component of event-related potentials (ERPs) has demonstrated considerable utility in the study of cognitive life-span changes, since it has been associated with basic informationprocessing mechanisms including attention and memory [33]. P3 is a large positive-going waveform with a posterior-parietal maximum amplitude and a peak latency of about 300-400 $\mathrm{ms}$ in young subjects, which has been obtained in the auditory, visual or somatic modality (for a recent review, see [19]). The visual P3 was shown to be significantly larger in amplitude and longer in latency than the auditory P3 [22,32]. In the ERP literature, P3 latency has been considered an indicator of the speed of cognitive processing associated with the selection of relevant stimuli; it is generally unrelated to response selection processes and independent of behavioral measures [26,30]. P3 amplitude has been considered as an index of the allocation of attentional brain resources to the voluntary selection of relevant stimuli (for a review, see [23]).

The results of several ERP aging studies employing auditory or visual paradigms demonstrated the existence of age-related changes in the latency, amplitude, and scalp distribution of this component. Specifically, there is general consensus that the peak latency of P3 progressively increases with age $[1,20,25,31,32,34,39,40]$. However, results on P3 amplitude changes with age are less consistent, especially for visual stimuli, since amplitudes were found to be unaffected by age in some studies [32], but reduced only at some electrode locations in others [31,34]. Other common finding in the literature is an age-related topographic alteration in the scalp distribution of P3, which becomes more anteriorly distributed and, thereby, more diffused or equipotential across the scalp with increasing age $[1,10-12,15,17,18,32,40]$. These age-related topographical changes have been interpreted as reflecting attentional alterations in older subjects, who would continue to utilize frontal processes for stimuli that have already been well-categorized $[1,10]$, (see [16] for a recent review). In parallel with these age-related changes in P3 scalp distribution, functional neuroimaging studies of aging have shown a paradoxical increase in the brain activation of older subjects during the execution of memory tasks, particularly in prefrontal areas $[6,28,35]$. It has been suggested that this age-related recruitment of atypical brain pathways might reflect a possible compensatory response (for reviews, see [3-5]). In keeping with this view, the 'compensation' hypothesis suggests that, in order to reach an adequate level of behavioral performance in a specific task, high performing older subjects would recruit different and wider areas of the brain, which are not activated by younger subjects (for reviews, see $[5,16,36]$ ). Alternatively, it has been suggested that this age-related increase in frontal activation could reflect an inefficient neural distribution of task-relevant cortical networks in older subjects, which was referred to as the 'dedifferentiation' hypothesis (see [3-5,28,36] for reviews). 
In our opinion, a fruitful way to shed new light on this debate would be to differentiate between performance levels in aging studies. Therefore, the aim of this study was to explore whether the P3 latency, amplitude, and scalp distribution differ in young and older subjects characterized as either high- or low-performers in a visual attention task. Since the typical oddball task is relatively easy, and performance in older subjects is usually almost perfect, we used a more demanding task. We tried to determine whether possible P3 differences between both subgroups of older subjects should be considered compensatory or inefficient. Indeed, if differences are due to a compensatory mechanism, highperforming older subjects should be expected to present a more widespread scalp distribution of P3 than young subjects. Alternatively, if the differences are due to a deficit or an inefficient neural activation, the scalp distribution changes should be observed in low-performing older subjects.

Because the previously described age-related P3 amplitude reductions may actually be due to: (a) the existence of more intertrial variability in P3 latency (i.e. latency jitter effect), (b) reduced P3 amplitudes in all trials, or (c) absence of P3 generation in some trials [26], we used a single-trial method to describe P3 fluctuations in addition to the conventional ERP averaging.

Finally, it should be noted that, whereas in most of previous studies the cognitive functioning of older subjects was defined by their performance on standardized neuropsychological tests [7], in our study the older subjects were characterized as either high- or low-performers according to their actual behavioral performance in the visual attention task. This allows us comparing these electrophysiological responses that are related to different actual performance levels among groups in the same task.

\section{Materials and methods}

\section{Subjects}

Ten young ( 7 females, age range: $22-38$, mean: $29.4 \pm 6.3$ years), and ten older subjects ( 5 females, age range: $58-67$, mean: $62.2 \pm 3.2$ years) were tested. All were healthy wellfunctioning subjects and had no history of neurological or psychiatric diseases. All subjects had normal or correctedto-normal vision. Older subjects performed the Spanish version (MEC-35) [27] of the Mini-Mental State Exam (MMSE) [13] and had normal scores (> 28). Informed consent was obtained from all subjects.

The older subjects were assigned to two groups on the basis of their behavioral performance in the visual attention task that was carried out during the recording of P3: a group of older subjects who performed as well as the young subjects (high-performing, $N=5$ ), and a group of older subjects who performed at a significantly lower level compared to the young subjects (low- 
performing, $N=5$ ). Reaction times (RTs) and performance levels confirmed that the older subjects assigned to the low-performing group, performed significantly worse than young and high-performing older subjects (see result section). Age and gender distributions were similar in both older groups (see Table 1).

\section{Stimuli and procedure}

Subjects sat in a comfortable armchair in an electrically shielded and sound-attenuated room at $61 \mathrm{~cm}$ viewing distance from a computer monitor. All stimuli were created, presented, and controlled using the Presentation software application (Neurobehavioral Systems, Inc., version 0.76). Afixation cross was presented continuously at the center of the monitor. The stimuli consisted of nine possible digits $(1,2,3,4,5,6,7,8$ or 9) in three different colors (red, green or blue) subtending $1.04 \circ \times 0.66^{\circ}$ of visual angle, which were equiprobably presented for $40 \mathrm{~ms}$ over the central fixation cross. Subjects were required to press a mouse button as quickly as possible in response to digits lower than five of any color. Thus, digits 1, 2, 3 and 4 of any color were the target stimuli.

Horizontal sinusoidal gratings differing in motion direction (4.13० visual angle, 20\% contrast, speed $1.95 \mathrm{deg} / \mathrm{s}$, spatial frequency $0.7 \mathrm{cycles} / \mathrm{deg}$ ) were also presented bilaterally at $10.7 \circ$ to the left and to the right from the fixation cross for $133 \mathrm{~ms}$. These gratings were presented in sequences of repetitive upward-drifting gratings (standard motion, $p=0.8$ ), which were occasionally replaced by downward drifting gratings (deviant motion, $p=0.2$ ) and were followed by a blankscreen ISI of $665 \mathrm{~ms}$. Subjects were presented with a block of 770 trials, from which 500 correspondedto unattended gratings and 270 to attended digits. All stimuli were presented with a stimulus onset asynchrony (SOA) of $798 \mathrm{~ms}$. Subjects were required to fix their gaze on the central cross and to pay attention to the digits, while ignoring the peripheral gratings. In a recent study, we reported the effects of normal aging on the preattentive processing of these unattended peripheral gratings [29] and here we only report the results on the central task.

\section{ERP recordings}

Continuous electroencephalogram (EEG) was recorded (bandpass $0.05-100 \mathrm{~Hz}, 500$ Hz/channel) with a NeuroScan system from 20 active electrodes (Fp1, Fp2, F3, Fz, F4, F7, F8, C3, Cz, C4, P3, Pz, P4, T3, T4, T5, T6, O1, O2, Oz), referred to the nose tip and grounded with an electrode at nasion. Vertical and horizontal eye movements were recorded bipolarly with additional electrodes placed above and below the left eye and at the outer canthi of both eyes, respectively. Electrode impedance was kept below $10 \mathrm{~K}$. 


\section{Data analysis}

Behavioral data analysis RTs were automatically on-line recorded for all subjects, and the performance level was calculated as the percentage of correct responses to target digits in the central visual task. Only RT values associated with correct responses were considered for data analysis. Mean RTs and percentages of correct responses were compared across groups using one-way analysis of variance (ANOVA) with Group (young, high-performing older, lowperforming older) as the between subjects factor.

\section{ERP data analysis}

Two types of analysis were performed on ERP data: conventional analysis of amplitude, latency and scalp distribution, and single-trial analysis.

\section{Conventional averaging}

The EEG was digitally filtered off-line with a $0.1-30 \mathrm{~Hz}$ bandpass filter, and epoched into periods of $1000 \mathrm{~ms}$ (100 ms pretarget and $900 \mathrm{~ms}$ post-target). Epochs exceeding $\pm 100 \mu \mathrm{V}$ and those containing horizontal or vertical eye movements, or incorrect responses were excluded from analysis. The EEG was averaged for the target digits in each group of subjects, separately. The P3 component was then measured as the maximum positive voltage peak between 300 and 600 ms poststimulus relative to the $100 \mathrm{~ms}$ baseline in each group of subjects. These amplitude values were subjected to mixed ANOVA with Group (young, high-performing older, low-performing older) as the between-subjects factor, and Localization (anterior, posterior), and Electrode (anterior: Cz, Fz, F3, F4, Fp1, Fp2; posterior: Pz, Oz, P3, P4, O1, O2) as the within-subject factors. The P3 latency values were determined with respect to the largest positive voltage at $\mathrm{Pz}$ electrode and compared across groups using one-way ANOVA with Group (young, highperforming older, low-performing older) as the between-subjects factor. Note that, in this study, we explored the age-related differences in $\mathrm{P} 3$ parameters along an anterior-posterior axis, so data on specific electrodes are not presented. An alpha level of 0.05 was used for all statistical tests. Degrees of freedom were corrected by the conservative Greenhouse-Geisser estimate when appropriate. Post hoc comparisons were performed using the Bonferroni adjustment for multiple comparisons.

Finally, in order to examine the scalp distribution of $\mathrm{P} 3$ and to explore in more detail the possible changes in the scalp distribution of P3 amplitude among groups, voltage maps were computed with the EEGLAB program [8], which plots topographic maps of EEG fields as a 2D circular view using cointerpolation on a fine Cartesian grid.

Single-trial analysis 
In order to visualize and describe more accurately the trial-to-trial variability in the amplitude and latency of P3 component during the task, EEG epochs to target digits associated with correct responses were subjected to single-trial analysis using the EEGLAB software [8] at Fz and $\mathrm{Pz}$ electrodes in each subject. In a first step, EEG Neuroscan data epoch files including only correct responses were imported via the EEGLAB toolbox under MATLAB environment. The specific channels of interest (Fz and $\mathrm{Pz}$ ) were then selected. Thereafter, the EEGLAB menu allowed us to sort data trials according to their occurrence in the experiment and, finally, to create ERP-image plots. The computed ERP-images consisted of two-dimensional colored rectangular representations of trial data, in which each horizontal line represents activity occurring in each single experimental trial. In these images the activity values are color-coded in left-to-right straight lines, with the changing color value indicating potential variations at each time point in the trial. Inspecting the adjacent single trials allowed us to explore the trial-by-trial P3 consistency, making possible to determine whether a P3 response was present in each individual trial, and to explore its moment-to-moment fluctuations in each group of subjects. Results of this single-trial analysis were compared with those of the conventional averaging.

\section{Results}

\section{Behavioral data}

RTs and percentages of correct responses are summarized in Table 1. There was a significant main effect of Group on mean RT $(F 2,17=5.67, P<0.013)$. Pairwise comparisons (Bonferroni) revealed that the mean RT of low-performing older subjects was significantly longer than that of young subjects $(P<0.011)$, with no significant differences between the mean RT of highperforming older and young subjects $(P=1)$, and between both low- and high-performing older subjects $(P=0.138)$. There was a significant main effect of Group on percentage of correct responses $(F 2,17=7.74, P<0.004)$. Pairwise comparisons revealed that performance accuracy was significantly lower for the low-performing older subjects than for the other two groups (young: $P<0.005$; high-performing older: $P<0.017$ ), with no significant differences between the performance levels of the latter two groups $(P=1)$ (see Table 1 ).

\section{ERP data}

Figure 1 shows ERPs to targets for young, high-, and lowperforming older subjects at anterior and posterior scalp locations. As can be seen, all three groups showed a clearly identifiable P3 component.

\section{P3 latency}


There were significant differences in P3 latency among groups $(F 2,17=12.24, P<0.001)$, revealing a significant latency increase in both older groups (high- and lowperformers). Pairwise comparisons revealed that P3 latency was significantly shorter for the young subjects than for the other two groups, independently of their performance level (high-performing older: $P<0.038$; low-performing older: $P<0.001)$, with no significant differences between both older groups $(P$ $=0.306)$.

\section{P3 amplitude and scalp distribution}

P3 scalp distribution differed significantly across groups (Localization x-Group interaction, $F 2,17$ $=5.23, P<0.017)$. Thus, as is apparent from Figure 1, low-performing older subjects showed a significant $\mathrm{P} 3$ amplitude reduction at posterior locations, compared to high-performing older and young subjects. Pairwise comparisons revealed that young and high-performing older subjects presented a significant difference of $\mathrm{P} 3$ amplitude between anterior and posterior scalp locations, with maximal posterior P3 amplitudes (young: $P<0.0001$; high-performing older: $P<0.001$ ), whereas P3 amplitude of low-performing older subjects did not differ significantly between anterior and posterior brain areas $(P=0.934)$. This topographical difference is illustrated in Figure 2, which displays the P3 mean amplitude values for each group at anterior and posterior locations. Voltage topographic maps (Figure 3) show a clear posterior positive focus of P3 in young and high-performing older subjects. As can be seen in the maps, there was no apparent difference in the pattern of voltage distribution between these two groups. However, in the maps corresponding to low-performing older subjects, we observed a marked reduction of the posterior P3 focus, giving rise to an apparently more uniform anterior-posterior P3 scalp dis tribution. These topographic data are consistent with the ANOVA results.

\section{Single-trial analysis of $P 3$}

As can be seen in Figure 4, the low-performing older subjects showed fewer trials with a clear P3 at Pz electrode than high-performing older and young subjects. Furthermore, even for trials in which P3 was visually detected at this electrode, the component was smaller in low-performing older subjects than in the other two groups. Moreover, in young and high-performing older subjects, a clear difference in the $\mathrm{P} 3$ variability between $\mathrm{Fz}$ and $\mathrm{Pz}$ electrodes can also be observed, showing larger P3 amplitudes at $\mathrm{Pz}$ electrode and more intertrial variability at $\mathrm{Fz}$ location. However, in the low-performing older subjects, there was no appreciable difference in P3 variability between these two electrodes. 


\section{P3 latency}

As expected, P3 latency increased with age, which is in accordance with many previous studies reporting P3 age-related delays $[1,20,25,31,32,34,39,40]$. Because P3 latency has been considered a valuable tool for studying the timing of cognitive processing (it reflects the time needed to perceptually identify, recognize, and select the target stimulus [26,30]), the age-related P3 latency prolongation has been interpreted in the literature as an evidence of cognitive slowing. In our study, all older subjects, even those who performed as well as the young subjects, showed a significant P3 latency prolongation. Thus, we can conclude that they showed a significantly slower processing speed than young subjects. This finding is consistent with previous reports of a progressive and generalized slowing of cognitive operations with advancing age [38].

Noteworthy, high-performing older subjects in our study showed a significant P3 latency delay, but not a significant RT prolongation, as compared to young subjects. In keeping with this finding, significant age-related increases in P3 latency without significant increases in RT were previously reported [14]. This may reflect that once a stimulus has been correctly selected as relevant, the response selection process is generated without delay. P3 latency has been shown to be relatively independent of the time required to select and execute a response, possibly measuring different aspects of stimulus processing. Thus, this result suggests that P3 latency would be more sensitive to normal aging than RT.

\section{P3 amplitude and scalp distribution}

In our study, we found changes in P3 amplitude that are associated with age and performance level in a visual attention task. A consistent finding in the literature has been an age-related change in P3 amplitude distribution across the scalp, which becomes more anteriorly oriented, and more equipotentially and uniformly distributed $[1,10-12,15,17,18,32,40]$. This distribution change implies that older subjects show a smaller P3 at posterior electrodelocations and a somewhat larger P3 at anterior locations than young subjects [20]. In our study, changes in P3 scalp distribution were observed only in low-performing older subjects relative to high-performing older and young subjects. Specifically, low-performing older subjects showed a significant P3 amplitude reduction at posterior, but not anterior, scalp locations, when compared to both other groups, who showed a posterior predominance of the target elicited P3.

The fact that the older subjects who performed better (high performers) showed a similar posterior-maximal P3 scalp distribution as young subjects, is in accordance with previous results [11]. Using an oddball task, Fabiani et al. [11] observed that, when compared to young subjects, older subjects showed a greater variability in the scalp location at which P3 was largest. Based on this finding, the authors divided their older sample into two groups: a group of older subjects who 
showed frontal-maximal P3 scalp distribution, and a group of older subjects who showed a posteriormaximal scalp distribution. They observed that the scalp topography of the older posterior-maximal group was similar to that of the young group. Moreover, they found that these older subjects with posterior-maximal P3 scalp distribution were less impaired in standardized tests of mental ability than those older subjects with frontally predominant P3s, although the formers were still functioning at a lower level than young subjects.

These age-related variations in P3 amplitude have been considered in the literature as an index of changes in the distribution of neural activity across the scalp. Accordingly, one could argue that low-performing older subjects showed less activity than young and high-performing older subjects in posterior brain areas. However, given that the electrical activity recorded by a scalp electrode may not have its origin in the brain area underneath the electrode, it is not possible to infer that the amplitude changes observed at posterior scalp electrodes are generated in the posterior brain areas. In this regard, there is general agreement that the scalp-recorded P3 component represents the summation of neural activity from several widely-distributed areas in the brain [21,37]. Thus, more precise inferences about P3 neural generators may be possible using neural imaging techniques with a better spatial resolution at the same time as ERPs are recorded. In this line, a recent fMRI study did localize the neural generators of the visual P3 component at parietal and inferior temporal areas, with contribution of both higher visual and supramodal association areas [2].

In the ERP literature, P3 amplitude has been also considered an indicator of the allocation of attentional brain resources (for a review, see [23]), being proportional to the amount of attentional resources employed to effectively select the stimuli that are relevant for a given task. Accordingly, the significant reduction of posterior P3 amplitude that we observed in the low-performing older subjects could suggest a possible reduction or decline in the allocation of attentional resources to the relevant stimuli. Nevertheless, although the age-related P3 amplitude changes have been considered reflecting functional visual attention deficits [25], the functional significance of amplitude measures is not yet completely understood [24], so that our results in this respect should be interpreted with caution.

As mentioned above, our data showed that the amplitude and scalp distribution of P3 to target stimuli were comparable between young and high-performing older subjects, showing maximal amplitudes at posterior locations. Thus, the striking finding of this study was the lack of an age related variation in the amplitude and scalp distribution of P3 component in the high-performing older subjects. A similar pattern of results was recently reported by Daffner et al. [7] who observed that, after controlling for a nonspecificage-related processing difference, the amplitude and scalp distribution of P3 were comparable among cognitively high-performing older, middle-aged, and 
young subjects. These data suggest that cognitively high-functioning older subjects may not show age-related differences specific to the processing of visual targets, as indexed by the visual P3 amplitude or scalp distribution.

\section{Single-trial analysis of P3}

Application of the single-trial method to ERP studies could provide more precise information about the characteristics and the dynamical changes of P3 than the conventional averaging method. To our knowledge, no previous studies have employed this method to explore the possible differences in P3 variability among groups of young and older subjects. Consequently, both conventional averaging and a single-trial method were used in the present study. Our singletrial data suggest that low-performing older subjects showed fewer trials with a clear P3 at Pz electrode than did high-performing older and young subjects, which suggests that low-performing subjects showed more waxing and waning of attention along the trials than both other groups. Furthermore, even in those trials with a clear P3 at Pz, the component was smaller in lowperforming older subjects than in both groups, which suggests a possible reduction in their attentional brain resources to be allocated to the correctly selected relevant stimuli. Such fluctuations in attention might contribute to the P3 amplitude reduction that was observed in the averaged waveforms of low-performing older subjects at posterior locations.

\section{Compensation versus dedifferentiation}

Remind that two divergent hypotheses were put forward in order to explain the paradoxical increase in frontal activation of older subjects. The compensation hypothesis suggests that highperforming older subjects would recruit different or wider brain areas than young subjects to compensate for the age-related neurocognitive decline and to successfully complete a specific task $[5,6,16,36]$. In contrast, the dedifferentiation hypothesis suggests that this age-related change occurs because older subjects become less able to recruit specialized neural mechanisms, reflecting a detrimental process in aging $[3-5,28,36]$.

In our study, the older subjects were assigned either to a high-performing or a low-performing group in order to explore whether they presented a different pattern of neural activation depending on their current performance during the task. The fact that the older subjects who performed better, recruited a similar neural network as young subjects, while older subjects who performed worse showed significant changes in their pattern of neural P3 activation, leads us to suggest that these low-performing older subjects might present a difficulty in engaging and activating the appropriate or specialized brain networks with the necessary extent to successfully perform the task. So, topographic maps and single-trial data corresponding to this group seemed to show a reduced recruitment in the specific posterior areas associated with $\mathrm{P} 3$, while the activity level at 
anterior areas was maintained. These data do not provide support for the compensation hypothesis since there were no observable differences in the pattern of neural activation of young and highperforming older subjects. Therefore, our results suggest the existence of a deficit mechanism in the low-performing older subjects, who showed an underactivation in posterior brain areas. It is possible that the poor performance of these subjects results from this underactivation of posterior brain regions. A similar pattern of results was obtained in a recent ERP memory study by Duarte et al. [9], who observed that high-performing older subjects exhibited neural correlates of memory recollection similar to those of the young subjects, while low-performing older subjects exhibited a different pattern of activity.

However, since the issue of compensation or dedifferentiation is fairly complex, we cannot reach definitive conclusions based solely on ERP data. We cannot also be sure whether the different pattern of neural activation observed in the low-performing subjects reflects functional deficits or different task-related strategies.

It is important to note that all the older subjects that participated in our study were healthy and intellectually active, and had normal scores in a measure of general cognitive function (MMSE). Nevertheless, the presence of undetected factors possibly causing the reduced cognitive functioning in low-performing older subjects cannot be ruled out. In addition, the fact that both older groups showed similar normative levels in the MMSE, but different performance levels in the visual attention task, emphasizes the need to clearly distinguish these cognitive functioning that are evaluated by means of normative tests those that are evaluated by means of actual behavioral measures.

\section{Conclusions}

Our findings indicate that the amplitude and scalp distribution of the visual P3 component significantly differ in older

subjects categorized in terms of their performance level in a visual attention task. These variations in P3 parameters between high- and low-performing older subjects might reflect differences in the efficiency of the visual processing of relevant stimulation between both older groups. In this regard, the fact that both older groups were similar in the MMSE mean score, mean age, and gender, suggests a specific decline in visual processing and not a generalized deficit in the lowperforming older subjects. According to the previous interpretations of age-related changes in the ERP literature, the significant RT and latency prolongation, and the posterior amplitude reduction of P3 component observed in low-performing older subjects, would indicate a decline in the intensity and speed of visual target processing, which implies a reduction in attentional resources in this older group. Furthermore, the altered P3 topographic distribution that we found in these 
subjects may suggest a difficulty in engage and activate the appropriate or specialized brain networks to a level that would be sufficient as to successfully perform the task.

Thus, these preliminary findings show that the patterns of cognitive deterioration in older subjects are complex, and that multifactorial processes may contribute to differential rates of cognitive aging. So, individual differences must be taken into account in future aging studies.

\section{Acknowledgements}

This research was supported by grants from the Spanish MEC (SEJ2004-01377) and Xunta de Galicia (PGIDT05PXIC21101PN). The authors are grateful to Prof. Jean-Michel Gu'erit and three anonymous reviewers for their valuable comments on an earlier version of the paper.

\section{References}

[1] Amenedo E, Díaz F. Aging-related changes in processing of nontarget and target stimuli during an auditory oddball task. Biol Psychol 1998;48(3):235—67.

[2] Bledowski C, Prvulovic D, Hoechstetter K, Scherg M, Wibral M, Goebel R, et al. Localizing P300 generators in visual target and distractor processing: a combined event-related potential and functional magnetic resonance imaging study. $J$ Neurosci 2004;24(42):9353-60.

[3] Buckner RL. Memory and executive function in aging and AD: multiple factors that cause decline and reserve factors that compensate. Neuron 2004;44(1):195-208.

[4] Cabeza R. Cognitive neuroscience of aging: contributions of functional neuroimaging. Scand J Psychol 2001;42(3):277-86.

[5] Cabeza R. Hemispheric asymmetry reduction in older adults: the HAROLD model. Psychol Aging 2002;17(1):85-100.

[6] Cabeza R, Anderson ND, Locantore JK, McIntosh AR. Aging gracefully: compensatory brain activity in high-performing older adults. Neuroimage 2002;17(3):1394-402.

[7] Daffner KR, Ryan KK, Williams DM, Budson AE, Rentz DM, Scinto LFM, et al. Age-related differences in novelty and target processing among cognitively high performing adults. Neurobiol Aging 2005;26(9):1283-95.

[8] Delorme A, Makeig S. EEGLAB: An open source toolbox for analysis of single-trial EEG dynamics including independent component analysis. J Neurosci Methods 2004;134:9_ 21. 
[9] Duarte A, Ranganath C, Trujillo C, Knight RT. Intact Recollection Memory in Highperforming Older Adults: ERP and Behavioral Evidence. J Cogn Neurosci 2006;18(1):33-47.

[10] Fabiani M, Friedman D. Changes in brain activity patterns in aging: the novelty oddball. Psychophysiology 1995;32(6): 579—94.

[11] Fabiani M, Friedman D, Cheng JC. Individual differences in P3 scalp distribution in older adults, and their relationship to frontal lobe function. Psychophysiology 1998;35(6):698-708.

[12] Fjell AM, Walhovd KB. P300 and neuropsychological tests as measures of aging: scalp topography and cognitive changes. Brain Topogr 2001;14(1):25—40.

[13] Folstein MF, Folstein SE, McHugh PR. "Mini-mental state". A practical method for grading the cognitive state of patients for the clinician. J Psychiatr Res 1975;12(3):189-98.

[14] Ford JM, Duncan-Johnson CC, Pfefferbaum A, Kopell BS. Expectancy for events in old age: stimulus sequence effects on P300 and reaction time. J Gerontol 1982;37(6):696-704.

[15] Ford JM, Pfefferbaum A. Event-related potentials and eyeblink responses in automatic and controlled processing: effects of age. Electroencephalogr Clin Neurophysiol 1991;78(5):361-77.

[16] Friedman D. Cognition and aging: a highly selective overview of event-related potential (ERP) data. J Clin Exp Neuropsychol 2003;25(5):702-20.

[17] Friedman D, Kazmerski V, Fabiani M. An overview of age-related changes in the scalp distribution of P3b. Electroencephalog Clin Neurophysiol 1997;104(6):498_-513.

[18] Friedman D, Simpson G, Hamberger M. Age-related changes in scalp topography to novel and target stimuli. Psychophysiology 1993;30(4):383-96.

[19] Hruby T, Marsalek P. Event-Related Potentials-the P3 Wave. Acta Neurobiol Exp 2003;63:55-63.

[20] Iragui VJ, Kutas M, Mitchiner MR, Hillyard SA. Effects of aging on event-related brain potentials and reaction times in an auditory oddball task. Psychophysiology 1993;30(1):10-22.

[21] Johnson R. On the neural generators of the P300 component of the event-related potential. Psychophysiology 1993;30(1):90-7. 
[22] Katayama J, Polich J. Auditory and visual P300 topography from a 3 stimulus paradigm. Clin Neurophysiol 1999;110(3):463—8.

[23] Kok A. Event-related-potential (ERP) reflections of mental resources: a review and synthesis. Biol Psychol 1997;45(1-3):19—56.

[24] Kok A. Age-related changes in involuntary and voluntary attention as reflected in components of the event-related potential (ERP). Biol Psychol 2000;54(1-3):107—43.

[25] Kutas M, Iragui V, Hillyard SA. Effects of aging on event-related brain potentials (ERPs) in a visual detection task. Electroencephalogr Clin Neurophysiol 1994;92(2):126-39.

[26] Kutas M, McCarthy G, Donchin E. Augmenting mental chronometry: the P300 as a measure of stimulus evaluation time. Science 1977;197(4305):792-5.

[27] Lobo A, Ezquerra J, Burgada FG, Sala JM, Seva A. El Mini- Examen Cognoscitivo: Un test sencillo y pr'actico para detectar alteraciones intelectuales en pacientes m'edicos. Actas Luso Esp Neurol Psiquiatr 1979;7:189—202.

[28] Logan JM, Sanders AL, Snyder AZ, Morris JC, Buckner RL. Underrecruitment and nonselective recruitment: dissociable neural mechanisms associated with aging. Neuron 2002;33(5):827-40.

[29] Lorenzo-L'opez L, Amenedo E, Pazo-Alvarez P, Cadaveira F. Pre-attentive detection of motion direction changes in normal aging. Neuroreport 2004;15(17):2633-6.

[30] McCarthy G, Donchin E. A metric for thought: a comparison of P300 latency and reaction time. Science 1981;211(4477): 77—80.

[31] Pfefferbaum A, Ford JM. ERPs to stimuli requiring response production and inhibition: effects of age, probability and visual noise. Electroencephalogr Clin Neurophysiol 1988;71(1):55-63.

[32] Pfefferbaum A, Ford JM, Wenegrat BG, Roth WT, Kopell BS. Clinical application of the P3 component of event-related potentials I. Normal aging. Electroencephalogr Clin Neurophysiol 1984;59(2):85-103.

[33] Polich J. Meta-analysis of P300 normative aging studies. Psychophysiology 1996;33(4):334-53.

[34] Polich J. EEG and ERP assessment of normal aging. Electroencephalogr Clin Neurophysiol 1997;104(3):244-56. 
[35] Reuter-Lorenz P, Jonides J, Smith EE, Hartley A, Miller A, Marshuetz C, et al. Age differences in the frontal lateralization of verbal and spatial working memory revealed by PET. J Cogn Neurosci 2000;12(1):174-87.

[36] Reuter-Lorenz P. New visions of the aging mind and brain. Trends Cogn Sci 2002;6(9):394-400.

[37] Ruchkin DS, Johnson R, Canoune HL, Ritter W, Hammer H. Multiple sources of P3b associated with different types of information. Psychophysiology 1990;27(2):157—76.

[38] Salthouse TA. The processing-speed theory of adult age differences in cognition. Psychol Rev 1996;103(3):403-28.

[39] Van der Lubbe R, Verleger R. Aging and the Simon task. Psychophysiology 2002;39(1):100-10.

[40] Verleger R, Neukater W, Kompf D, Vieregge P. On the reasons for the delay of P3 latency in healthy elderly subjects. Electroencephalogr Clin Neurophysiol 1991;79(6):488 — 502. 
Table 1 Sample characteristics, mean RTs and percentage of correct responses (mean \pm SD)

\begin{tabular}{|c|c|c|c|}
\hline & Young $(N-10)$ & Older-high $(N-5)$ & Older-low $(N-5)$ \\
\hline Mean age and range & $29.4 \pm 6.3 ; 22-38$ & $62.2 \pm 2.7 ; 58-64$ & $62.2 \pm 4 ; 59-67$ \\
\hline Gender (f/m) & $7 / 3$ & $3 / 2$ & $2 / 3$ \\
\hline Mean MUSE score ${ }^{a}$ & Not tested & $33.4 \pm 2.3^{a}$ & $33.2 \pm 2.5^{\mathrm{a}}$ \\
\hline RT (ms) & $463.7 \pm 39$ & $489.8 \pm 33$ & $564.5 \pm 91$ \\
\hline Performance level $(\%)$ & $99 \pm 1$ & $98.9 \pm 1.1$ & $94.2 \pm 4.5$ \\
\hline
\end{tabular}

Note that the Spanish version of the MUSE is scored on a maximum of 35 .

Anterior Locations

Fpl

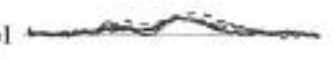

F3<smiles>C=CCC=CCC</smiles><smiles>C#CCCCCCC</smiles><smiles>COc1ccccc1</smiles>

$\mathrm{F} z$

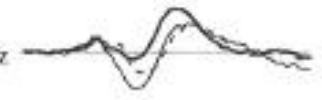<smiles>C#CC1CCCCC1</smiles>

Posterior Locations<smiles>C#CC1CCCCC1CCP</smiles><smiles>C=CCC=CCC=O</smiles>

160 \& $100200300509600 \leqslant 62702800 \leqslant 0$

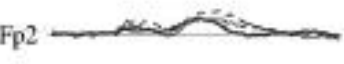

F4<smiles>C#CC=CC=CC</smiles><smiles>CC1CCCCC1CP</smiles><smiles>C#CCCOCCO</smiles>

Older low-porforming

Figure 1 Grand-average ERP waveforms elicited by targets in young, high-and low-performing older subjects at the six anterior and posterior electrodes that were subjected to statistical analysis. Note the P3 latency increase in both older groups and the P3 amplitude reduction in low-performing older subjects at posterior locations. 
P3 amplitude at anterior and posterior scalp locations

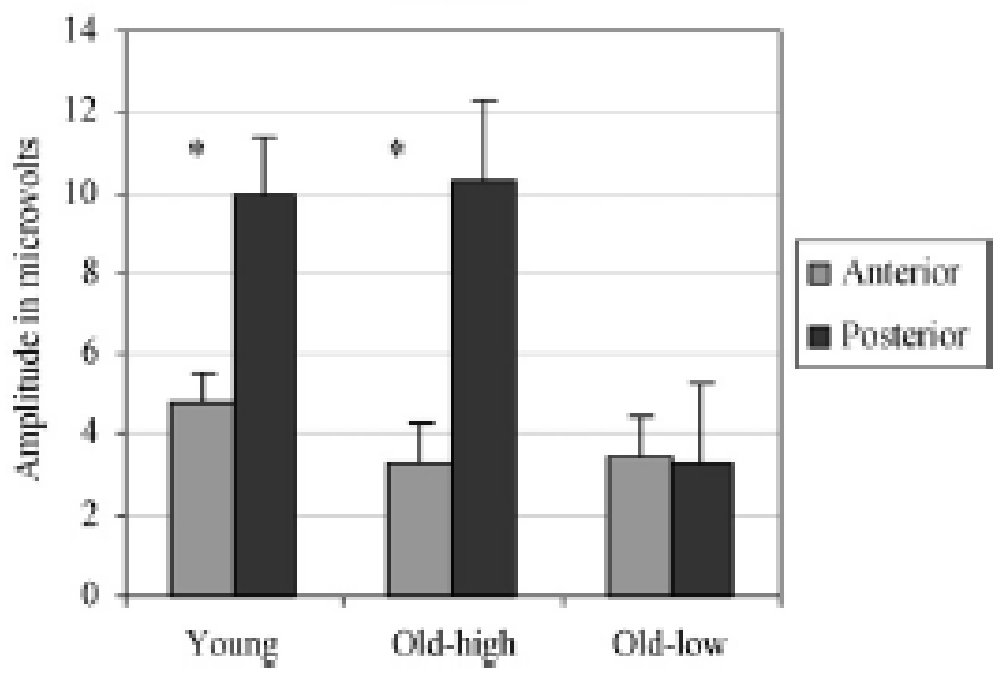

Figure 2 Distribution of P3 amplitude mean values at anterior (gray bars) and posterior (black bars) locations for each group of subjects. Significant differences of P3 amplitude between anterior and posterior locations can be observed in young and high-performing older subjects (marked with an asterisk), but not in low-performing older subjects.
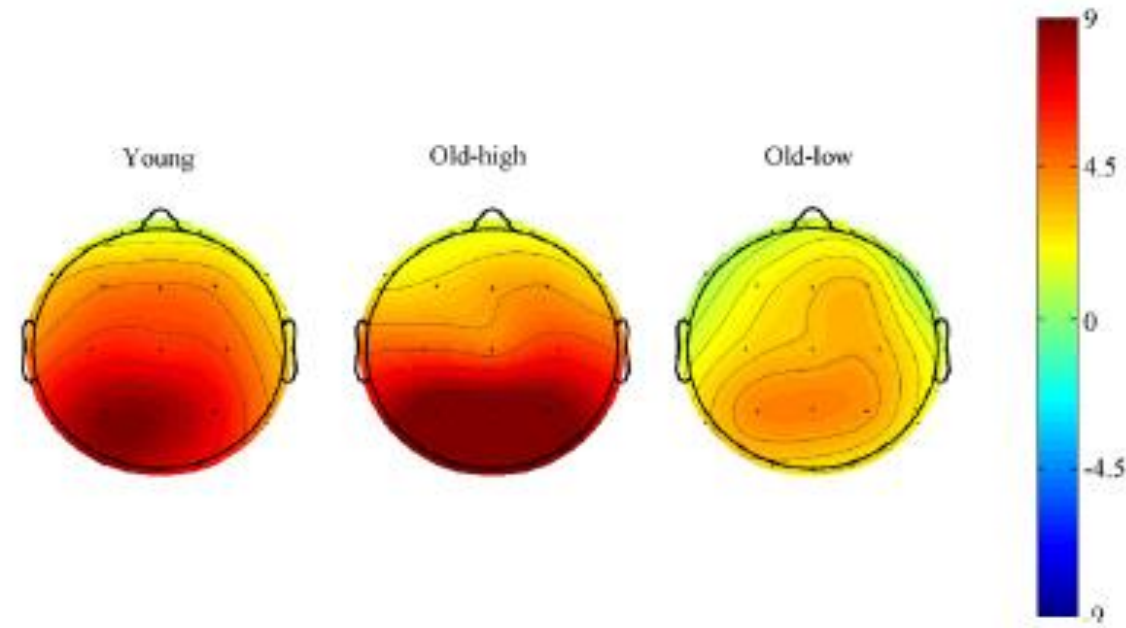

Figure 3 Voltage topographic distribution of P3 amplitude in young, high- and low-performing older subjects. The color scales depict P3 amplitudes in $\mu \mathrm{V}$ (hot colors indicating high amplitudes). Note that the P3 posterior positive focus that is apparent in young and high-performing older subjects is almost absent in low-performing older subjects. 

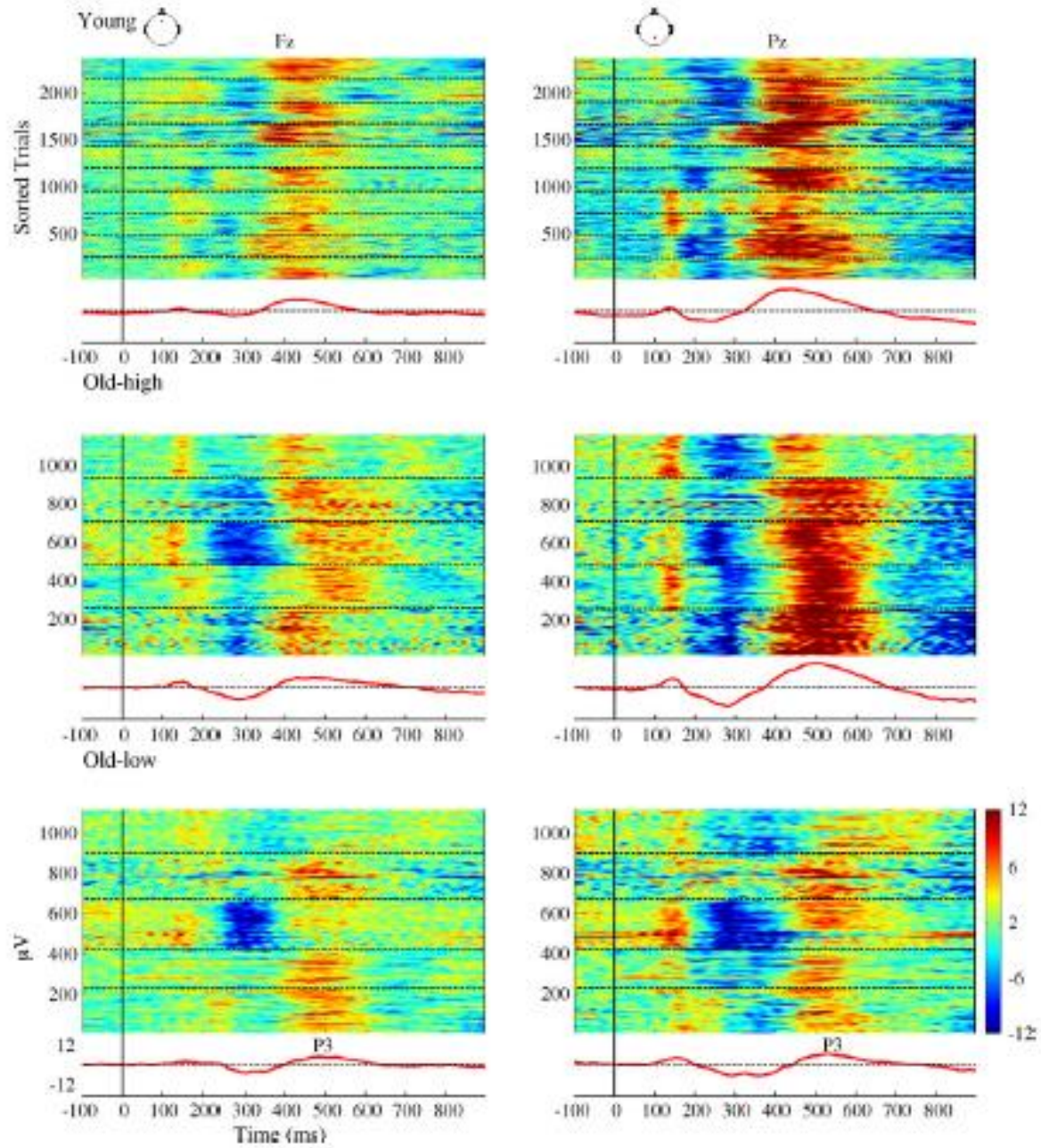

Figure 4 ERP images of single-trials recorded at $\mathrm{Fz}$ and $\mathrm{Pz}$ electrodes, time-locked to onsets of correctly selected visual target stimuli (left vertical line). Each horizontal line represents activity occurring in a single experimental trial. Voltage variations are color-coded (see color bar at bottom-right). The trace below the ERP image shows the ERP average of the imaged data epochs and contains the characteristic P3 component. The head plot containing a red dot indicates the scalp position of the electrode whose data are imaged. Single-trial activity corresponding to each subject is separated by horizontal dashed black lines. Trials are sorted in order (bottom-to-top) of their occurrence in the experiment. 